\title{
Sputter-Induced Topography on Magnesium During Ion Beam Milling Surface Preparation
}

\author{
Shirin Kaboli, Hendrix Demers, Nicolas Brodusch and Raynald Gauvin \\ Department of Mining and Materials Engineering, McGill University, Montréal, Canada.
}

For any microstructural observations in a scanning electron microscope (SEM), appropriate specimen preparation is essential such that a mechanical damage free, oxide free, clean and reasonably flat surface is achieved. Such surface can be achieved using chemical or, more commonly, electrochemical polishing techniques after mechanical polishing steps [1]. However, successful electrochemical polishing involves proper selection of variables such as chemical composition and temperature of electrolyte, voltage and time which, sometimes, can be tedious particularly for polycrystalline and multiphase specimens with strained microstructure [1,2]. Ion beam milling is used as an alternative preparation technique to replace electrochemical polishing technique. Using appropriate milling conditions for each type of material, an oxide free, clean and reasonably flat surface can be achieved. In addition, rate of material removal from surface can be controlled using the milling parameters. The drawbacks of ion milling include ion beaminduced structural and physical changes on the surface which can alter secondary electron (SE) or backscattered electron (BSE) yield in SEM. A variety of sputter-induced topographical features have been reported which depend on both specimen conditions and ion beam parameters [3].

Ion beam milling was applied on a bulk polycrystalline pure $\mathrm{Mg}$ specimen, using a Hitachi IM-3000 flat ion milling system. Ion beam milling was performed at near normal ion beam incidence with respect to the specimen surface. High magnification SE micrographs of the specimen surface after ion beam milling are shown in Figure 1. A wide range of topographical features was observed in grains with different crystallographic orientations. Such observations include cones (Fig. 1a), etch pits (Fig. 1b), bubbles (Fig. 1c) and periodic and wavelike ripples (Fig. 1d). Formation of all topographical features was attributed to different localized sputtering yield relative to the surrounding area. For the same ion beam milling parameters (i.e. energy, flow, and angle of incidence), the sputtering yield depends on specimen parameters (i.e. crystallographic orientation, purity, defects and pre-existed topography), and on the surrounding environment (i.e. presence of contamination and neighboring material). Low magnification SE and BSE micrographs obtained from the same area showed different contrast variations. Grains with high angle misorientation, subgrains with low angle misorientation, low angle and high angle grain boundaries were observed in SE micrograph (Fig. 1e). However, only grains with high angle misorientation and high angle grain boundaries were observed in BSE micrograph (Fig. 1f). Generated surface topography during ion beam milling resulted in different SE yield from misoriented areas at low magnification. However, surface topography perturbs channeling contrast and leads to undetectable BSE yield differences between grains of different orientation. As a result, misoriented domains could not be differentiated in BSE micrograph.

Sputter-induced topography has an adverse and large effect on SE and BSE micrograph in SEM. The type and extent of topography development for an arbitrarily chosen specimen is not accurately predicted. Ion beam parameters can be optimized to control the extent of topography development. However, the optimal ion beam milling parameters depend on the specimen conditions and experimentation is necessary to determine the best operating conditions for surface preparation. 


\section{References:}

[1] A. J. Wilkinson and P. B. Hirsch, Micron 28 (1997), pp. 279-308.

[2] C. Ulrich, F. Schwarz, A. Franke, T. Marr, D. Rafaja, L. Kruger, J. Freudenberger, Pract Mettallog 49 (2012), pp. 290-304.

[3] A. W. Czanderna, T. E. Madey, C. J. Powell, Beam Effects, Surface Topography, and Depth profiling in Surface Analysis, New York: Plenum Press, 1998.
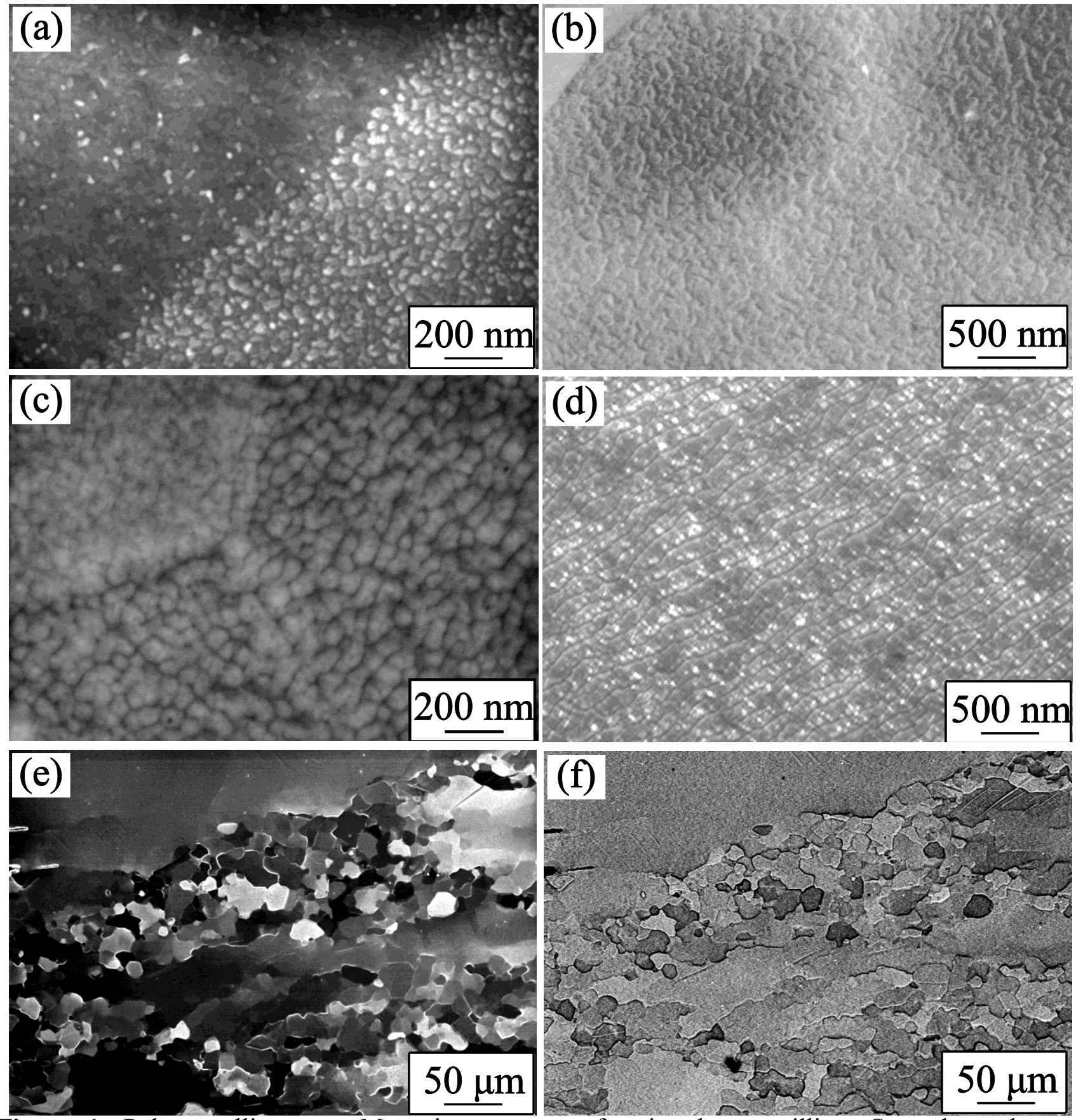

Figure 1. Polycrystalline pure $\mathrm{Mg}$ microstructure after ion beam milling. Secondary electron micrograph of (a) cones, (b) etch pits, (c) bubbles and periodic and wavelike ripples. (e) Secondary electron and (f) backscattered electron micrographs at low magnification. 